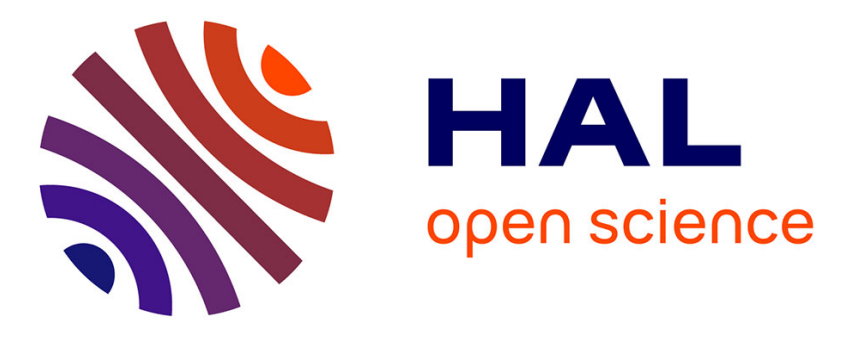

\title{
Practical Indoor Localization using Ambient RF
}

Ye Tian, Bruce Denby, Iness Ahriz Roula, Pierre Roussel, Rémi Dubois, Gérard Dreyfus

\section{To cite this version:}

Ye Tian, Bruce Denby, Iness Ahriz Roula, Pierre Roussel, Rémi Dubois, et al.. Practical Indoor Localization using Ambient RF. 2013 IEEE International Instrumentation and Measurement Technology Conference (I2MTC), May 2013, United States. pp.1125-1129, 10.1109/I2MTC.2013.6555589 . hal-00961295

\section{HAL Id: hal-00961295 \\ https://hal.science/hal-00961295}

Submitted on 24 Mar 2014

HAL is a multi-disciplinary open access archive for the deposit and dissemination of scientific research documents, whether they are published or not. The documents may come from teaching and research institutions in France or abroad, or from public or private research centers.
L'archive ouverte pluridisciplinaire HAL, est destinée au dépôt et à la diffusion de documents scientifiques de niveau recherche, publiés ou non, émanant des établissements d'enseignement et de recherche français ou étrangers, des laboratoires publics ou privés. 


\section{Practical Indoor Localization using Ambient RF}

\author{
Ye Tian, Bruce Denby (SM'99) \\ SIGMA Laboratory and \\ Université Pierre et Marie Curie \\ Paris, France \\ ye.tian@etu.upmc.fr, \\ denby@ieee.org
}

\author{
Iness Ahriz \\ LAETITIA/CEDRIC Laboratory \\ Conservatoire National des Arts et \\ Métiers \\ Paris, France \\ iness.ahriz@cnam.fr
}

\author{
Pierre Roussel, \\ Rémi Dubois (M'09), \\ Gérard Dreyfus (F'12) \\ SIGMA Lab, ESPCI ParisTech \\ Paris, France \\ firstname.lastname@espci.fr,
}

\begin{abstract}
The article presents a simple, practical approach for indoor localization using Received Signal Strength fingerprints from the GSM network, including an analysis of the relationship between signal strength and location, and the evolution of localization performance over time. Support Vector Machine regression applied to very high dimensional fingerprints does not reveal any smooth functional relationship between fingerprints and position. Classification using Support Vector Machines however provides very good results on discriminating different rooms in an indoor environment, albeit with performance that degrades over time. Transductive inference, introduced as a means of updating models to overcome degradation over time, provides hints that accurate indoor localization can be achieved by applying classification methods to cellular Received Signal Strength fingerprints, performance robustness being maintained via model updating and refining.
\end{abstract}

Keywords-localization; indoor; fingerprint; transductive support vector machine

\section{INTRODUCTION}

Indoor localization systems are an important extension to Location Based Services (LBSs), for assisted living scenarios, tracking of Alzheimer's patients, and in more general situations [1]. As GPS receivers are unable to function in indoor environments, a variety of indoor localization strategies have been proposed to try to tackle this challenging task [2-11]. Methods based on the measurement of Received Signal Strength (RSS) in RF networks such as Wi-Fi and Bluetooth networks, for example, have proven to be effective [2-6]. However, time and labor intensive deployment and maintenance of these networks is a drawback that reduces the impact of these techniques.

Aside from these specially deployed networks, indoor localization based on ambient radiotelephone networks, such as GSM and CDMA, has also been studied [8-10]. In the past few years, methods based on the use of RSS fingerprints acquired from large numbers of GSM channels have appeared promising $[10$, and references therein]. Recent results also suggest that an appropriately programmed standard cellular mobile phone can provide a simple, inexpensive solution for accurate room-level indoor localization [11].

The studies proposed in [10-11], however, were preliminary for two reasons. First, they did not attempt to explore the distribution of measured RSS values, or to discover a functional relationship between RSS and position in indoor environments. Secondly, no prescription was made for correcting for RSS drifts over time, which is a well-known challenge in RSS based systems, particularly for long-range signals [12]. Indeed, due to shadowing, multipath and environmental effects such as building geometry, network traffic, presence of people, and atmospheric conditions, RSS is expected to be nonlinear with distance, non-Gaussian, and time varying [2], which can lead to performance degradation over time.

In this article, after an initial description of the data collection procedures used (section II), we turn our attention to seeking a functional relationship between GSM RSS and position, using Support Vector Machine (SVM) regression. The results, in fact, presented in section III, show that no such relationship exists for the indoor environments tested, indicating that interpolation/extrapolation schemes based on RSS measurements at a small number of points as in [10] will not be viable for localization. .

On the other hand, because of the local nature of shadowing and multipath effects, RSS fingerprints acquired over entire rooms can potentially be useful for room-level classification, as proposed in [11]. The scheme is presented in section IV, using data collected during "random walks" that explore an entire area. This section also presents long term tests showing that the performance of our RSS based indoor localization method degrades over time. We introduce transductive inference, which uses new, incoming unlabeled data to update SVM classifiers as a means of reducing performance degradation caused by RSS drift. The use of small amounts of new labeled data as a model update scheme is also explored here. Overall conclusions and some perspectives appear in section $\mathrm{V}$.

\section{MEASUREMENT SitES AND DATASETS}

Two types of datasets were collected, for regression and classification experiments, respectively, both recorded in a 4th floor laboratory building (steel frame, concrete and plaster walls) in central Paris, France (Fig. 1). The first set, which we shall call the regression set, was collected in a single room (office 7) using so-called machine-to-machine, or M2M, GSM/GPRS modules [13], which can be driven using standard and manufacturer-specific AT modem commands. We used 8 identical modules, with nominally identical specifications and technical parameters. During data collection, the M2M 


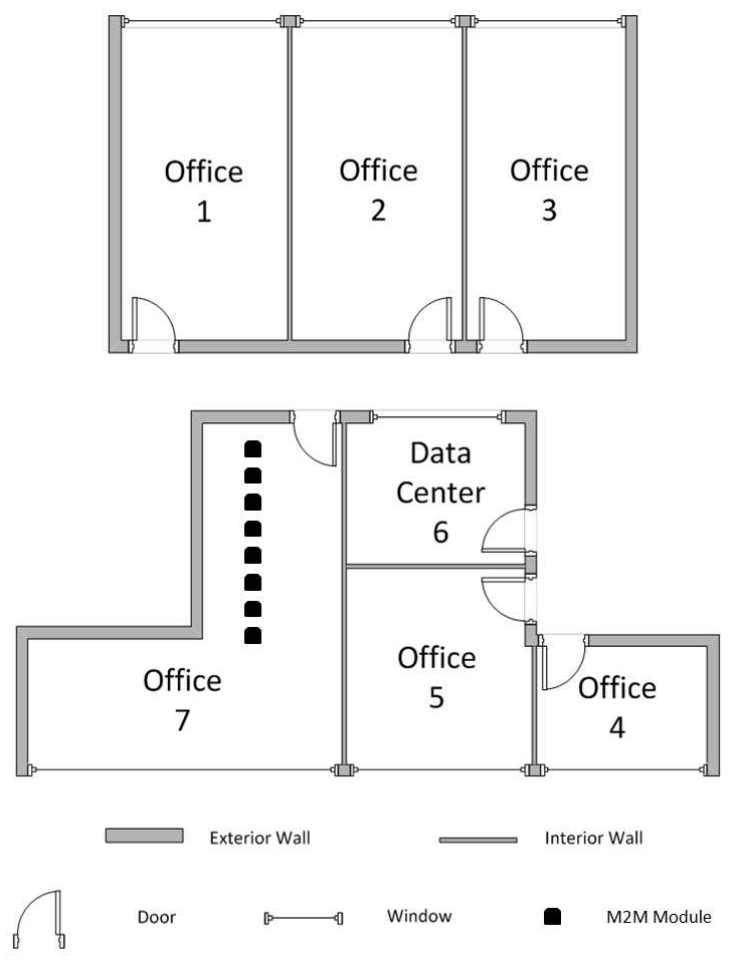

Figure 1. Layout of the laboratory where the datasets were recorded

modules were placed at fixed positions, in a line, spaced at an interval of $0.6 \mathrm{~m}$, as illustrated in Fig. 1. A total of 600 GSM scans for each module were recorded over 5 working days. Each scan contains the RSS of all 548 carriers in the GSM900 and GSM1800 bands, and consists of RSS values ranging in value from $-108 \mathrm{dBm}$ to $-40 \mathrm{dBm}$. All the scans were labeled manually with location from $0 \mathrm{~m}$ to $4.2 \mathrm{~m}$ indicating where the scan was made.

The second dataset, which will be used for SVM classification, was collected in 7 rooms of the laboratory as described in [11]. The data acquisition device in this case is a Sony-Ericsson mobile phone with embedded scanning software, which is able to obtain a scan of the entire GSM900 and GSM 1800 bands in about 300 milliseconds. Scans were recorded in each of the 7 rooms and manually labeled with the corresponding room numbers during "random walks". Four datasets were recorded during weekends over a period of six months in the same setting, hereafter called $S 1$ (5000 scans in each room), S2 (2000 scans), S3 (1000 scans) and S4 (1000 scans).

\section{POSITION FROM RSS}

It was shown in [10] that the classification of RSS vectors recorded at fixed points works quite well. The objective here is to see if a regression method allows to measure locations at intermediate positions between the fixed points by finding a functional relationship between location and RSS in this indoor environment.

\section{A. SVM Regression Algorithms}

Since the number of variables is very large (548 carriers) and the size of the training set is relatively limited, SVM regression was deemed appropriate because of its built-in regularization mechanism [16].

Consider a given dataset $\left\{\left(\mathbf{x}_{1}, y_{1}\right),\left(\mathbf{x}_{2}, y_{2}\right), \cdots,\left(\mathbf{x}_{n}, y_{n}\right)\right\}$, where $\mathbf{x}_{i}$ is the fingerprint vector at location $i$ and $y_{i}$ is the coordinate of the location (assuming that 1-D localization is performed as described above). There exists a variety of Support Vector Regression (SVR) techniques, serving different purposes. $\varepsilon-S V R$, which was used in our experiments, aims to find a parameterized function $f(\mathbf{x}, \theta)$ such that prediction errors $\left\|y_{i}-f\left(\mathbf{x}_{i}, \theta\right)\right\|$ do not exceed a given value $\varepsilon$ for all elements of the training set, and, at the same time, is as regular as possible, i.e. does not wiggle unnecessarily [14]. Assume that we are looking for a linear relationship between the RSS fingerprint and the location. The function has the form:

$$
f(\mathbf{x}, \theta)=\mathbf{w} \cdot \mathbf{x}+b
$$

where $\theta=\left[\begin{array}{ll}\mathbf{w} & b\end{array}\right]^{T}$. The parameters are sought as solutions to the constrained optimization problem:

$$
\begin{array}{ll}
\text { minimize } & \frac{1}{2}\left\|\mathbf{w}^{2}\right\| \\
\text { subject to } & \left\|y_{i}-\left(\mathbf{w} \cdot \mathbf{x}_{i}-b\right)\right\| \leq \varepsilon
\end{array}
$$
form

The optimal solution, if it exists, can be shown to be of the

$$
f(\mathbf{x})=\sum_{i=1}^{n} \alpha_{i} y_{i}\left(\mathbf{x}_{i} \cdot \mathbf{x}\right)+\alpha_{0}
$$

where $\alpha_{0}$ and all the $\alpha_{i}$ are solutions of a constrained quadratic optimization problem.

If such a solution does not exist, slack variables $\zeta_{i}$ and $\zeta_{i}^{*}$ can be introduced to relax the constraints, allowing some examples of the training set to be predicted with an error larger than $\varepsilon$. The problem becomes:

$$
\begin{aligned}
\operatorname{minimize} & \frac{1}{2}\left\|\mathbf{w}^{2}\right\|+C \sum_{i=1}^{n}\left(\zeta_{i}+\zeta_{i}^{*}\right) \\
\text { subject to } & \left\{\begin{array}{c}
y_{i}-\mathbf{w} \cdot \mathbf{x}_{i}-b \leq \varepsilon+\zeta_{i} \\
\mathbf{w} \cdot \mathbf{x}_{i}+b-y_{i} \leq \varepsilon+\zeta_{i}^{*} \\
\zeta_{i}, \zeta_{i}^{*} \geq 0
\end{array} \quad \forall i\right.
\end{aligned}
$$

where $C$ is a hyperparameter called "regularization constant".

If we want to look for a non-linear relationship between the RSS fingerprint and the location, non-linear regression can be realized by first performing a nonlinear transformation of the variables that defines a more suitable feature space, in which linear regression is performed. The final solution is in the form

$$
f(\mathbf{x})=\sum_{i=1}^{n} \alpha_{i} y_{i} K\left(\mathbf{x}_{i}, \mathbf{x}\right)+\alpha_{0}
$$

where $K(.,$.$) is called the kernel function. In our experiments,$ linear and nonlinear regression (using Gaussian and Polynomial kernels) were performed, using the Spider toolbox [15].

\section{B. Results}

The results of SVRs are estimated through the mean squared localization error $\frac{1}{8} \sum_{k=1}^{8} \sqrt{\frac{1}{600} \sum_{i=1}^{600}\left[y_{k}-f\left(\mathbf{x}_{i k}, \boldsymbol{\theta}^{-k}\right)\right]^{2}}$, 
TABLE I. REGRESSION RESULTS

\begin{tabular}{|c|c|}
\hline Regression Method & Mean Squared Localization Error \\
\hline Linear LS-Regression & $2.3 \mathrm{~m}$ \\
\hline Linear SVM Regression & $1.8 \mathrm{~m}$ \\
\hline Polynomial SVM Regression $(d=5)$ & $1.3 \mathrm{~m}$ \\
\hline Gaussian SVM Regression & $2.4 \mathrm{~m}$ \\
\hline
\end{tabular}

where $y_{k}$ is the position of measuring device $k, \mathbf{x}_{i k}$ is the RSS vector measured during scan $i$ taken at location $k$, and $\boldsymbol{\theta}^{-k}$ is the parameter vector found by training from the data pertaining to all locations except location $k$.

The results for linear and non-linear regressions are shown in Table I. The soft margin parameter $C$, polynomial degree $d$ and Gaussian kernel parameter $\sigma$ were selected through crossvalidation. Results from linear least squares regression (LSRegression) are also given for comparison.

As shown in the table, the mean positioning error of all the regression methods is so large as to be unexploitable. The regression error is approximately equal to the average distance between the 8 locations, meaning that no linear or non-linear relationship between RSS and the position in a small indoor environment is evident. This appears to rule out using full-band RSS GSM vectors obtained in this way to interpolate between fixed positions in an indoor localization method.

\section{ROOM LEVEL CLASSIFICATION AND RSS DRIFT}

An alternative scheme, first introduced in [11], is to perform room-level classification based on RSS measurements made during "random walks". In this case, we use classifiers to perform room-level indoor localization, where each room is a class. Data-driven classification problems are often solved in two stages: off-line training; and on-line testing. In the off-line training stage, discriminant functions are determined using training data and known labels, while in the on-line testing stage, a new test fingerprint is presented to the classifier and given a label based on the discriminant functions.

The RSS-based room level SVM classifiers in [10] gave good results when training and test data were obtained over the same one-month period. Here, we explore the time dependence of the "random walk" approach, proposing transductive inference to continuously adjust a discriminant function with newly collected unlabeled data, in order obtain an updated classifier.

\section{A. SVM Classifier}

SVM classifiers were used in our experiments, since they are deemed appropriate to deal with the high dimensional RSS fingerprints for the same reasons as described above for SVRs.

Consider a set of $n$ examples of items belonging either to class $\mathrm{A}$ or class $\mathrm{B}$, each example being described by a $p$ dimensional vector $\mathbf{x}_{i}$. Further assume that the examples are linearly separable, i.e. that there exists a hyperplane of equation $f(\mathbf{x})=0$ that separate all examples without error: $f\left(\mathbf{x}_{i}\right)>0$ for all examples $i$ belonging to class $\mathrm{A}$ and $f\left(\mathbf{x}_{i}\right)<0$ otherwise. It can be proved that $f(\mathbf{x})$ can be written under the form

$$
f(\mathbf{x})=\sum_{i=1}^{n} \alpha_{i} y_{i}\left(\mathbf{x}_{i} \cdot \mathbf{x}\right)+\alpha_{0}
$$

where the $\alpha_{i}(\mathrm{i}=0 \cdots n)$ are parameters whose values are estimated from the examples; $y_{i}=+1$ if example $i$ belongs to class $A$ and $y_{i}=-1$ otherwise.

If the examples are not linearly separable, a "soft-margin" approach can be used to reduce the complexity of the classifier by introducing slack variables $\zeta_{i}$ and performing a tradeoff between accuracy of classification of the training examples and ability to generalize; the price to pay is the introduction of a "regularization" constant $C$ whose value must be chosen appropriately.

\section{B. Transductive SVM Classifier}

Transductive SVMs take unlabeled test examples into account and adjust the separating surface to separate both training examples and test examples with maximum margin. For a linearly separable data case, this leads to the following optimization problem [17]:

$$
\begin{array}{ll}
\operatorname{minimize} & \frac{1}{2}\left\|\mathbf{w}^{2}\right\| \\
\text { subject to } & \left\{\begin{array}{l}
y_{i}\left(\mathbf{w} \cdot \mathbf{x}_{i}+b\right) \geq 1 \\
y_{j}^{*}\left(\mathbf{w} \cdot \mathbf{x}_{j}^{*}+b\right) \geq 1
\end{array} \quad \forall i, j\right.
\end{array}
$$

where $x_{j}^{*}$ is the unlabeled data and $y_{j}^{*}$ is the label corresponding to $x_{j}^{*}$ given by TSVMs. Therefore, minimization must be performed with respect to $\mathbf{w}, b, \mathbf{x}_{j}{ }^{*}$ and $y_{j}{ }^{*}, j=1 \ldots N$, by contrast to standard SVMs where minimization must be performed with respect to $\mathbf{w}$ and $b$ only. To be able to handle non-separable data, slack variables $\zeta_{i}$ are introduced as in standard SVM classifiers. Algorithms for solving this optimization problem are described in $[17,18]$.

The transductive SVMs used in our study, were implemented using SVM ${ }^{\text {light }}$ [19].

\section{Results, and Comparison to "Re-Training"}

Experimental results are shown in Table II. The performance is presented as the percentage of correctly classified test examples. The results shown on the first row were published in [11] and reproduced here for comparison. The first 100 unlabeled test examples of each room in $S 1, S 2$ and $S 3$ sets were used for TSVM training to adjust the model, the remaining examples were used for testing. In SVM classifiers, we use the model trained on set $S 1$ to test the test data in sets $S 2, S 3$ and $S 4$. Only linear one-vs-all multi-class scheme was used because this method gave the best performance when testing on set $S 1$.

As can be seen in Table II, when building the classifier with set $S 1$ and testing it on sets $S 2, S 3$ and $S 4$ taken in different time periods, the performance varies dramatically, from about $94 \%$ for "fresh" data (S1 set), down to as low as 32\% (S4 set). However, by using only 100 new unlabeled test examples of each room with the TSVM, a substantial amount of the lost performance can be recovered, with accuracies up to $78.4 \%$. 
TABLE II. COMPARISON OF SVM AND TSVM

\begin{tabular}{|c|c|c|c|}
\hline Test Set & Training Set & SVM & TSVM \\
\hline$S 1$ & $S 1$ & $94.2 \%$ & - \\
\hline$S 2$ & $S 1$ & $60.4 \%$ & $78.4 \%$ \\
\hline$S 3$ & $S 1$ & $39.7 \%$ & $58.8 \%$ \\
\hline$S 4$ & $S 1$ & $32.3 \%$ & $49.6 \%$ \\
\hline
\end{tabular}

TABLE III. RESULTS OF RE-TRAINING THE MODEL

\begin{tabular}{|c|c|c|c|}
\hline Training Data & Test Data & Algorithm & Result \\
\hline $\begin{array}{c}\text { The first } 100 \\
\text { scans of } S 3\end{array}$ & $\begin{array}{c}\text { The last } 900 \\
\text { scans of } S 3\end{array}$ & Linear one-vs-all & $83.3 \%$ \\
\hline $\begin{array}{c}\text { The first } 100 \\
\text { scans of } S 4\end{array}$ & $\begin{array}{c}\text { The last } 900 \\
\text { scans of } S 4\end{array}$ & Linear one-vs-all & $77.4 \%$ \\
\hline
\end{tabular}

The TSVM approach is interesting because it presents a way of recovering some of the performance loss due to RSS drift, at the cost only of obtaining some recent unlabeled RSS measurements. In practice, such data might be obtained from scans performed on the handsets of users of the localization system, but without the need to manually label the data. Though the improvement obtained with the TSVM is still not sufficient, the results nevertheless suggest that any scheme that keeps the classifier model "current", by tracking the evolution of the RSS values, should be of interest to us.

This hypothesis is supported by Table III, which presents the results of training a new classifier "from scratch" based on a small number of new labeled scans of each room. In this scenario, "hand" labeling of the update data is necessary, but could perhaps be performed by specially designated employees, or by volunteers in exchange for some "reward" (i.e., crowdsourcing). The table shows that even if the current model is too outdated to give good performance, the $S 3$ and $S 4$ sets for example, it can be trained using only a small amount of labeled data and give substantially improved performance.

\section{CONCLUSIONS AND PERSPECTIVES}

In a study of ambient RSS distribution in an indoor environment using SVM regression, no smooth functional relationship could be discovered between GSM RSS and position for the indoor environment tested, implying that interpolation-based techniques are not likely to be successful. The use of ambient GSM RSS-based classifiers trained with data collected throughout the areas of rooms, however, presents a viable alternative, and experimental results show that the percentage of correct room labeling can be up to $94 \%$ if the model is used before significant RSS drift sets in. In order to cope with performance degradation caused by RSS drift over time, transductive inference was introduced to update the SVM classifiers with new unlabeled data. When tested on data sets collected over 6 months, this approach proved capable of restoring a significant part of the lost performance. The use of small amounts of current labeled data to create "current" room classifiers also appears to be a promising approach, even if performance still needs to be improved.
In future work, we will continue to strive for a continuously updatable, high-performance indoor room classifier. We also intend to investigate the use of W-CDMA network data in our measurements.

\section{ACKNOWLEDGMENT}

The authors wish to acknowledge the support of the China Scholarship Council.

\section{REFERENCES}

[1] A. Küpper, Location-Based Services: Fundamentals and Operation, John Wiley \& Sons, New York, NY, USA, 2005.

[2] AM. Ladd, KE. Bekris, A. Rudys, LE. Kavraki, DS. Wallach, "On the feasibility of using wireless ethernet for indoor localization," IEEE Trans on Robotics and Automation. vol. 20, no. 3, pp.555-559, June 2004.

[3] Y. Zhou, C.L. Law, Y.L. Guan, and F.P.S. Chin, "Indoor Elliptical Localization Based on Asynchronous UWB Range Measurement", IEEE Trans. on Instrumentation and Measurement, vol 6, issue 1, pp.248-257, January 2011

[4] Q. Yang, S. Jialin Pan, V. Wenchen Zheng, "Estimating location using Wi-Fi," IEEE Intelligent Systems, vol. 23, no. 1, pp.8-13, January/February 2008.

[5] S-H. Fang, T-N. Lin, P-C. Lin, "Location fingerprinting in a decorrelated space," IEEE Trans on Knowledge Data Engineering. vol. 20, no. 5, pp.685-691, May 2008.

[6] S-H. Fang, T-N. Lin, "Indoor location system based on discriminantadaptive neural network in IEEE 802.11 environments," IEEE Trans on Neural Network, vol. 19, no. 11, pp.1973-1978, Nov 2008.

[7] S-H. Hong, B-K. Kim, D-S Eom, "Localization algorithm in wireless sensor networks with network mobility," IEEE Trans on Consumer Eectronic. vol. 55, no. 4, pp.1921-1928, Nov 2009.

[8] V. Otsason, A. Varshavsky, A. LaMarca, E. de Lara, “Accurate GSM indoor localization," in Proceedings of the 7th International Conference on Ubiquitous Computing, pp.141-158, Tokyo, Japan, September 2005.

[9] W. ur Rehman, E. de Lara, S. Saroiu, "CILoS, a CDMA indoor localization system," in Proceedings of the 10th International Conference on Ubiquitous Computing, pp.21-24, Seoul, South Korea, September 2008.

[10] Y. Oussar, I. Ahriz, B. Denby, G. Dreyfus, "Indoor localization based on cellular telephony RSSI fingerprints containing very large numbers of carriers", EURASIP J. Wireless Comm. and Network. 2011, 2011:81.

[11] Y. Tian, B. Denby, I. Ahriz, P. Roussel, G. Dreyfus. "Fast, HandsetBased GSM Fingerprints for Indoor Localization," In Proceedings of the The 9th International Symposium on Wireless Communication Systems, pp. 641-645, Paris, France, August, 2012.

[12] VW. Zheng, EW. Xiang, Q. Yang, and D. Shen. "Transferring localization models over time," In Proceedings of the 23rd National Conference on Artificial Intelligence, vol. 3, pp. 1421-1426.

[13] Telit GM862-GPS module, [Online]: http://www.telit.com/en/products/ gsm-gprs.php

[14] AJ. Smola, and B. Schölkopf, "A Tutorial on Support Vector Regression," 1998.

[15] The Spider, [Online]: http://people.kyb.tuebingen.mpg.de/spider/

[16] N. Cristianini, J. Shawe-Taylor. Support Vector Machines and Other Kernel-Based Learning Methods, Cambridge University Press, 2000

[17] T. Joachims, "Transductive Inference for Text Classification using Support vecter Machines," International conference on Machine Learning, pp. 200-209, Bled, Slovenia, June 1999.

[18] Y. Chen, G. Wang, and S. Dong, "Learning with Progressive Transductive Support Vector Machine," IEEE International Conference on Data Mining, Maebashi city, Japan, December 2002.

[19] SVMlight Support Vector Machine, [Online]: http://svmlight. joachims .org. 